\title{
Experimental Study: A LQI-Based Ranging Technique in ZigBee Sensor Networks
}

\begin{abstract}
Ranging technology which estimates the distance between two communicating wireless nodes has been widely used as a necessary component in localization solutions for wireless sensor networks (WSN). LQI (Link Quality Indicator) is a metric introduced in IEEE 802.15.4 that measures the error in the incoming modulation of successfully received packets that pass the CRC (Cyclic Redundancy Check). Because of low system cost and less computational complexity, LQI-based ranging techniques are increasingly applied in Zigbee sensor networks. However, due to the environmental affects and electronic noise generated by hardware, raw LQI data could not be directly aligned with distances. To eliminate errors in LQI data and obtain higher ranging accuracy, we design and evaluate a novel LQI-based ranging technique which includes three essential data processing components: pre-correction, error compensation and mixed regression analysis. First, anchor nodes with known locations are used in pre-correction process to correct LQI measurements against the empirical regression function obtained from historical data. Then, error compensation is applied to eliminate the intrinsic error in LQI data. Finally, ranging results are refined by the mixed regression analysis. The proposed ranging technique is implemented and evaluated on a Zigbee sensor prototypeTarax. Experiment results show that the average ranging error is less than $1 \mathrm{~m}$, confirming that the proposed technique is able to achieve higher ranging accuracy and suitable for localization applications in WSN.
\end{abstract}

Keywords: Link quality indicator (LQI); ranging techniques; Zigbee; wireless sensor networks; distance estimation. 


\section{Introduction}

Wireless sensor network (WSN) consists of spatially distributed autonomous sensors and are often randomly deployed in a large area for environmental monitoring, target tracking and security surveillance. In these applications, it is necessary to accurately orient the nodes with respect to a global coordinate system in order to report data that is geographically meaningful. Therefore, localization in WSN has been an active area for decades.

To achieve localization in WSN, some algorithms rely on pre-positioned nodes while others may require expensive specially-designed hardware. Some algorithms need a way of performing off-line computation, while others can do all calculations on sensors in real time. Localization is still an challenging and exciting field, with new algorithms, hardware, and applications being developed at a feverish pace. Generally, there are two major localization techniques in the literature: range-based and range-free algorithms (1). Since range measurements between nodes usually contain errors, a node's location can only be estimated. Localization algorithms dependent on distance measurements are sensitive to ranging errors. Range-free algorithms can overcome this problem, however, they perform poorly in irregular networks (2). This paper aims at improving ranging accuracy by desiging a novel LQI-based ranging technique for practical localization applications.

In this work, we closely investigated the relationship between LQI data and the distances being measured. From experiment results, we discovered that LQI data could be affected by other wireless networks, e.g. the WiFi networks (nearby the test sites) can cause interference to Zigbee networks. In addition, we found that LQI data are intensively correlated to distances when the distances are less than 3 meters, i.e. within this range, LQI gradually decreases when distance increases. Based on the above-mentioned observations, we first introduce a pre-correction algorithm to reduce the errors in LQI data by including reference nodes. Combing with the error compensation and mixed regression analysis, an new ranging method is established so that the error in distance estimation is minimized to an acceptable level.

\section{Related Work}

There are numbers of ranging techniques in wireless sensr networks including time of arrival (TOA), received signal strength indication (RSSI), time difference of arrival (TDOA), angle of arrival (AOA), etc. Some of these technologies may need special hardware, e.g. TDOA uses ultrasonic devices (3), AOA (4) requires antenna arrays. TOF does not need additional hardware and can calculate the distance between a sensor and an anchor node based on round-trip time (RTT) of packets. However, to obtain a higher ranging accuracy, lots of computing resources are needed to process huge amount of RTT samples $(5 ; 6)$. Moreover, due to the low signal-to-noise ratio(SNR), multi-path fading and hardware noise, huge errors are expected in RTT measurements (7).

\subsection{Received Signal Strength Indication (RSSI)}

RSSI has been widely used in wireless link quality assessments, distance measurements, and localization algorithms. An early system called RADAR (8) uses RSSI fingerprints to estimate a node's location. In (9), an approximated ranging technique by eliminating the mean error in RSSI was presented.

There are several works trying to enhance ranging accuracy based on RSSI data, e.g. Gauss model is used in (10), and (11) proposed a recursive filtering algorithm using weighted average with smooth factor map RSSI signal. Split range technique (12) is used to estimate approximate distance and able to predict the path of a moving target. There are other studies on CC2420 (13), which use standard deviation of RSSI and packet loss to estimate distance.

Previous studies have shown that reflection, scattering and other physical properties have severe impacts on RSSI measurements (14). Although (15) introduced an adequately adjusted RSSI measurements, the instability of RSSI measurement is always a problem. There are also approaches using artificial neural network (16) and RSSI fingerprint (17) to address the issues of environmental impact on RSSI.

\subsection{Link Quality Indicator (LQI)}

Recently, a new type of metric measuring wireless link was provided on Zigbee devices. The new metric is called Link Quality Indicator (LQI) which could be used for distance estimation (18).

LQI is a metric used to estimate the link qualities of RF transmissions. As defined in IEEE802.15.4, LQI is calculated by the physical layer and provides signal strength and link quality information to network and above layers after every data frame is received. LQI is relevant to the successful reception of data frames, i.e. higher LQI indicates better link quality and lower data loss rate.

For example, in the MC13224v chipset developed by Freescale, the value of LQI is an integeral multiple of 3 , ranging from $0 \mathrm{x} 00$ to $0 \mathrm{xFF}$ where $0 \mathrm{x} 00$ corresponds to $100 \mathrm{dBm}$ and $0 \mathrm{xFF}$ corresponds to $-15 \mathrm{dBm}$. The formula of computing LQI in MC13224v is as follows:

$$
L Q I(d B m)=\left(\frac{L Q I(d e c)}{3}-100\right)
$$

In the API provided by MC13224v, the function MLMELinkQuality () can be used to return a pointer referring to a 8-bit LQI value.

LQI has a wider range than RSSI, so it has a better resolution. When wireless devices are close to each other,

Copyright (C) 2008 Inderscience Enterprises Ltd. 
the LQI data have less deviation and mean-square (19). In (20), an experimental study was performed by using both RSSI and LQI in estimating distance between sensors. The results in (20) indicate that when LQI increases, the probability of correctly estimating distance is increasing. In (21), RSSI/LQI with fuzzy logic and TBM classification methods are used. The authors claimed that they can receive $80 \%$ ranging accuracy when devices are less than 4-meter between each other, but errors spread beyond 4 meters.

\section{Towards LQI-Based Ranging}

To completely understand whether and how LQI can be used in ranging techniques, we first designed and developed a wireless Zigbee sensor prototypeTarax. Creating a new prototype is necessary because commercial off-the-shelf (COTS) Zigbee devices may not meet our specific needs. In addition, COTS devices may not support user customization, which makes system debugging almost impossible. The Tarax node has a master chip MC13224v developed by Freescale, which contains a $2.4 \mathrm{GHz}$ RF transceiver, a 32-bit ARM7 processor, and an IEEE 802.15.4-compliant MAC protocol. Antenna is also an important component in getting consistent LQI readings. In experiments, only one type of antenna is used, i.e. a single-ended $50 \mathrm{Ohm}$ antenna with $0 \mathrm{dBi}$ antenna gain. In all experiments, we use two Tarax WSN nodes: one as the receiver and the other as the transmitter. Both of them are placed at the same height $(0.5 \mathrm{~m}$ from the ground). We fix the receiver's location and move the transmitter away from the receiver with a step size of $0.5 \mathrm{~m}$. There is no obstacle between these two nodes.

\subsection{Environmental Impacts on LQI}

To fully understand the environmental impacts on LQI of this particular type of Tarax node, we conduct several tests in different environments including a open field (concrete), a soil ground, a grass land, and an indoor environment.

As shown in Figure 1, we collected a set of LQI data at different atmospheric pressures (957Pa, 956Pa and $963 \mathrm{~Pa}$ ) when Tarax nodes are placed on a concrete open field. The figure shows that atmospheric pressures has no notable affect on LQI data. We also conduct experiments in different humidity and temperature, and no significant affect on LQI are observed. When environmental setting is fixed, similar LQI data are observed. However, the LQI data from different environment are quite different, indicating environmental impact on LQI could not be ignored.

\subsection{Inteference from Other Wireless Network}

Figure 2 shows a 24-hr continuous measurements of LQI data in an indoor environment. The experiment

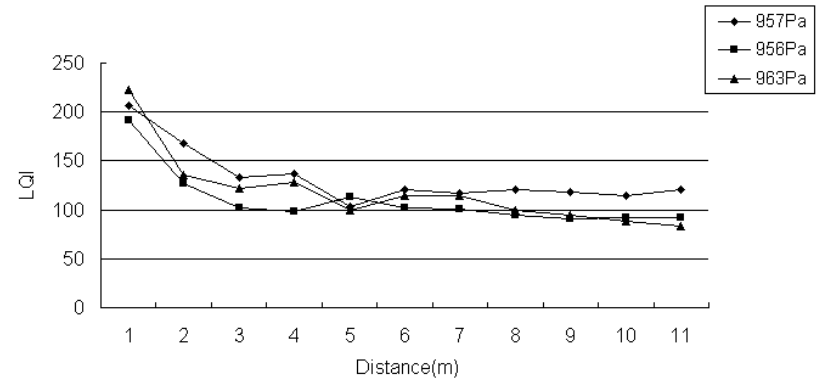

Figure 1 Illustration of Atmosphere Impact on LQI

was conducted during a weekday starting from 11:00AM to 11:00AM the next day. The figure shows that LQI data somehow correlated to human activities. In other words, LQI data fluctuate from 11:00AM to 10:00PM, but become stable from 10:00PM to 9:00AM. Because there is no human intrusion during tests, one possible explanation of LQI fluctuation is co-channel interference with WiFi signals. Due to the unpredictable nature of data traffic from other intefering networks, randomness in LQI data are expected to be seen.

\subsection{Correlation between LQI and Distance}

To investigate whether LQI data are correlated to distances, we conduct experiments in an open concrete field. The distances between transmitter and receiver nodes increase from $0 \mathrm{~m}$ to $40 \mathrm{~m}$ with a step size of $1 \mathrm{~m}$. At each distance, 100 LQI data are collected, and the average of these data is plotted in Figure3.

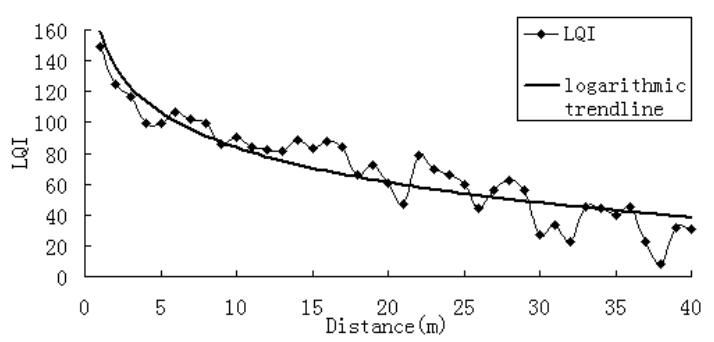

Figure 3 Correlation Between LQI and Distance within 40 Meters

As shown in Figure 3, although LQI data decline as distances increase, the plotted curve is not smooth, indicating a weak correlation between LQI data and distances. When distances are less than 10m, LQI data decrease faster with fewer fluctuations. However, more oscillations are observed when distances is greater than $10 \mathrm{~m}$. Overall, when distances increase, more LQI errors occur and the correlation between LQI and distances become more complicated. Therefore, The proposed ranging technique in this paper only works when devices are close to each other (i.e. within 10 meters). 


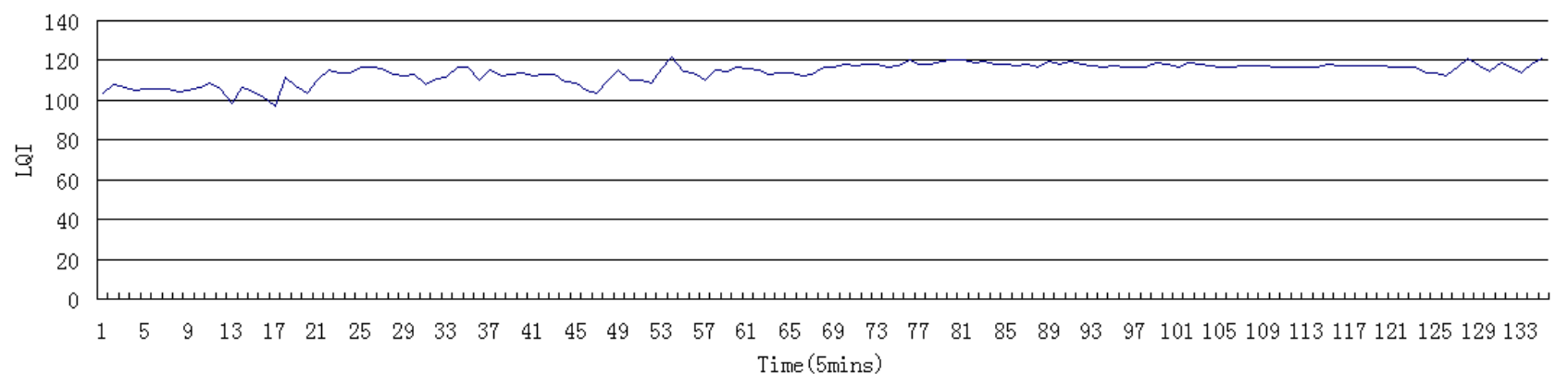

Figure 2 Illustration of the Impact of Human Activities on LQI

\subsection{Intrinsic Errors in LQI}

In Figure 3, we also notice that LQI data slightly increase when the distance changes from $4 \mathrm{~m}$ to $5 \mathrm{~m}$. To further understand this issue, we conduct several other experiments with different experiment setup parameters in various environments, and results from all scenarios show the same phenomenon. Thus, we conjecture that data fluctuation occurring from 4 to 5 meters may be caused by intrinsic errors in the hardware MC13224v.

Although data fluctuation are observed in Figure 3, generally, LQI data decrease when distances increase, which is true for all experiments we conducted. After analyzing all experiment results, we discover that fluctuations in different experiments follow a similar pattern. This important observation implicitly reveal that LQI data could be used for distance estimation given the intrinsic errors being identified and eliminated.

\section{LQI-Based Ranging Technique}

To realize a practical ranging technique, the LQIbased ranging algorithm must overcome the following challenges: 1) it must be resilient to the change of experimental environments; 2) it must be able to detect and eliminated the intrinsic errors in LQI data; and 3) due to unpredictable channel interference, randomness of LQI data is expected, and hence it must be resilient against the variation of LQI. We propose a novel ranging technique to meet these challenges via three key algorithms: pre-correction, error compensation, and mixed regression analysis.

\subsection{Empirical Regression Function}

Because LQI data are affected by many factors, an effective way to identify the relationship between LQI and distance is to conduct regression analysis on huge amount of data. By analyzing LQI data, an empirical regression function can be built to reflect the correlation between LQI data and distances. As shown in Figure 4, an logarithmic and polynomial fitting functions are shown based on the LQI data collected from our experiments.

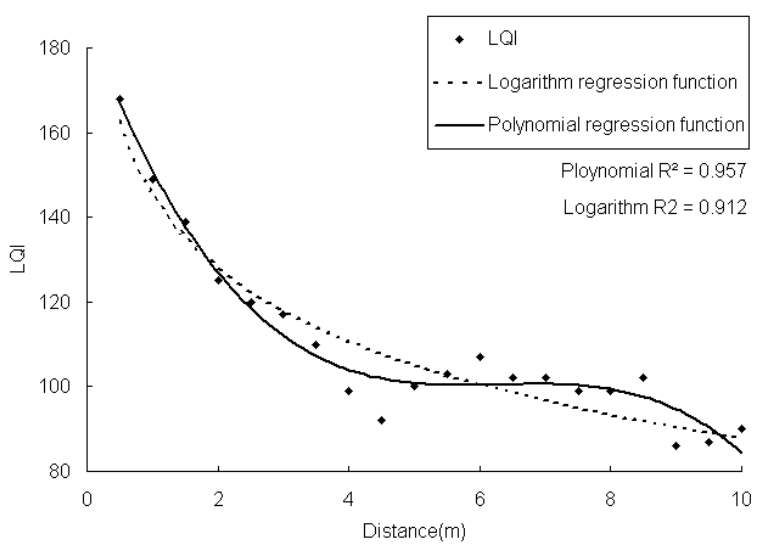

Figure 4 Illustration of the Empirical Regression Functions

In Figure 4, the logarithmic and polynomial functions have $R^{2}<0.9$, which means both of them are very close to the real LQI-and-distance curve. Since the polynomial function gives better results, we choose polynomial regression function to estimate the distances between nodes.

\subsection{Pre-Correction Algorithm}

As we discussed in Section 3.1, although atmospheric pressure (as well as humidity and temperature) do not have notable impact on LQI data, differences in the regression functions of various environments still exist. As shown in Figure 5, we conduct two experiments at the same location on different days, and the curves of "day1" and "day2" are the regression functions corresponding to the data collected in these two days, respectively. The other curve is the empirical regression function obtained by analyzing large amount of historical data. At the same distance, LQI data from different experiments ("day1" and "day2") show differences from the empirical curve. However, the trends of regression functions are similar, which means it is possible to build an accurate regression function by pre-correcting LQI data.

To eliminate minor differences between the actual and empirical regression functions, we make use of anchor nodes (with known locations). The basic idea of the pre-correction algorithm is to find the differences 


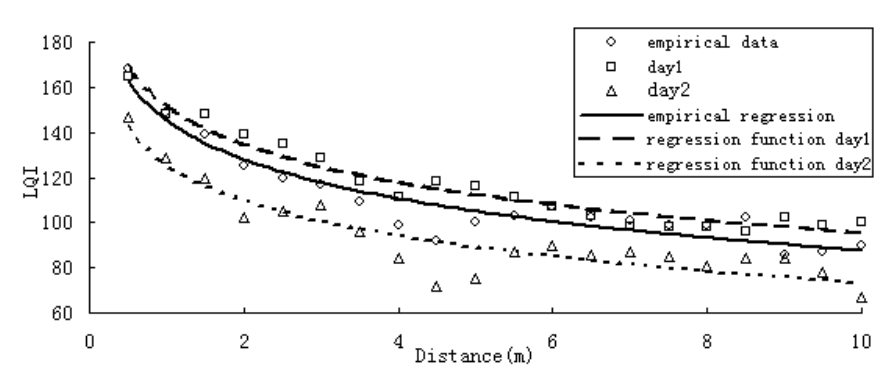

Figure 5 LQI Differences Between the Actual and Empirical Regression Functions

between the empirical regression function and the one corresponding to the current experiment data. When new LQI data are collected, they will be adjusted based on these differences to closely match the empirical regression function. There are two ways to eliminate the differences: 1) using the average differences of LQI to perform a unified correction, and 2) using the differences of similar LQI to conduct a grouped correction. We will first describe how to obtain LQI differences and then the error elimination approaches.

As shown in Figure 6, anchor nodes are denoted as $A_{0}, A_{1}, \ldots, A_{k}$, the distances from $A_{0}$ to $A_{1}, A_{2}, \ldots, A_{k}$ are $d_{1}, d_{2}, \ldots, d_{k}$, the target node is $M$ whose location needs to be estimated. The LQI data and corresponding distances can be grouped into tuples as $\left\langle L Q I_{1}^{0}, d_{1}>\right.$ $,<L Q I_{2}^{0}, d_{2}>\ldots,<L Q I_{k}^{0}, d_{k}>$. Since the reference node $A_{0}$ can be any nodes in $\left\{A_{1}, A_{2}, \ldots, A_{k}\right\}$, we use a general form $<L Q I_{1}, d_{1}>,<L Q I_{2}, d_{2}>\ldots,<$ $L Q I_{k}, d_{k}>$ to denote the LQI data measured by a anchor node with other $k-1$ anchor nodes. Comparing $L Q I_{i}$ to the LQI value from the empirical regression function at the distance $d_{i}$, differences $\delta_{i}$ between them can be computed. Let's use $L Q I(i)$ to denote the LQI measured by the target node $M$ from node $A_{i}$.

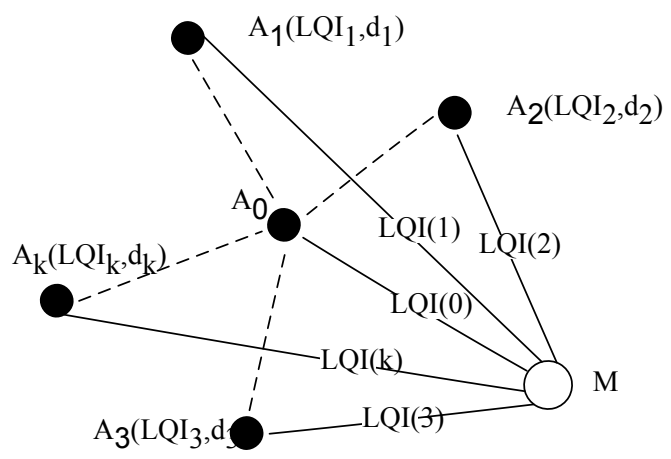

Figure 6 Illustration of the Pre-Correction Process

If the values of $\delta_{i}$ are similar, we use the average of $\delta_{i}$ to correct the LQI data $\{L Q I(i)\}$. The average LQI difference is computed as follows:

$$
\bar{\delta}=\frac{\sum_{i=1}^{k} \delta_{i}}{k},\left|\delta_{i}-\delta_{j}\right| \leq \epsilon, i, j \in\{1, \ldots, k\}
$$

where $\epsilon$ is an adjustable parameter that can be configured according to application requirements. If $\left\{\delta_{i}\right\}$ show large deviations, unified correction may not be applicable as correction would result in even greater errors. Under this circumstance, $\left\{\delta_{i}\right\}$ data should be grouped, and the number of groups is determined by the distribution of $\delta_{i}$.

As shown in Figure $7,\left\{\delta_{i}\right\}$ are divided into three groups, and $\left\{\delta_{i}\right\}$ in each group has a small deviation. Within each group, unified correction is used to correct the LQI data $\{L Q I(i)\}$. The correction value of LQI in each group is calculated as follows:

$$
\bar{\delta}_{m}=\frac{\sum_{i=1}^{k_{m}} \delta_{i}}{k_{m}},\left|\delta_{i}-\delta_{j}\right| \leq \epsilon_{m}, i, j \in\left\{1, \ldots, k_{m}\right\}
$$

where $m$ is the index of each group, $\bar{\delta}_{m}$ is the correction value of $L Q I$ in group $m, k_{m}$ is the number of $\left\{\delta_{i}\right\}$ in group $m, \epsilon_{m}$ is the maximum difference of $\delta_{i}$ in group that can be determined by the applications.

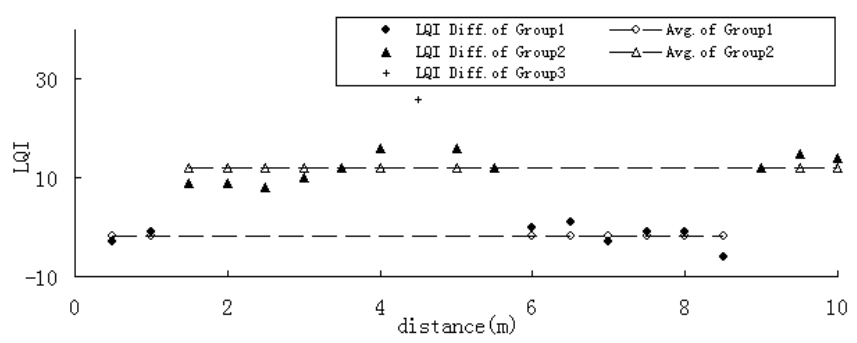

Figure 7 Example of the Group Correction Process

Figure 7 shows the grouped $\delta_{i}$ collected in the experiment "day2". In the figure, there are three different groups of $\delta_{i}$, the $\bar{\delta}_{m}$ of each group is shown as a dash line.

Due to the fluctuation of LQI, similar LQI may correspond to different distances. Besides, $L Q I(i)$ may not have a corresponding tuple (with same distance) in $\left\{\delta_{i}\right\}$. To address these issues, the grouped correction works as follows.

Step1 We first define an LQI interval $\Delta$, e.g. 10dBm. Then, $L Q I_{i}$ in $\left\langle L Q I_{i}, d_{i}>\right.$ can be grouped into a set $B$ as follows. Starting from the $\min \left\{\delta_{i}\right\}$, group all $L Q I_{i}$ less than $\left(\min \left\{\delta_{i}\right\}+\Delta\right)$ into a subset $B_{0}$. Update $\left\{L Q I_{i}\right\}$ to $\left\{L Q I_{i}\right\}-\left\{B_{0}\right\}$, and find the next group in the same way. Then the average LQI correction value $\bar{\delta}_{m}$ of each group $m$ can be computed accordingly.

Step2 If $L Q I(i)$ appears in a certain group $B_{m}$, then goes to Step3; otherwise, switch to Step4.

Step3 If $L Q I(i)$ exist in only one group $B_{m}$, then $\bar{\delta}_{m}$ can be used to correct $L Q I(i)$ to $L Q I(i)+\bar{\delta}_{m}$. If $L Q I(i)$ appears in two groups, e.g. $B_{m}$ and $B_{n}$, then $\frac{\bar{\delta}_{m}+\bar{\delta}_{n}}{2}$ will be used to correct $L Q I(i)$.

Step 4 If $L Q I(i)$ does not appear in any group $B_{m}$, then the $L Q I_{j}: \min \left\{\left|L Q I_{j}-L Q I(i)\right|\right\}$ is chosen and goes to Step3. If there are more than one $L Q I_{j}$ existing in more than one group, then the average $\bar{\delta}_{m}$ of these group will be used to correct $L Q I(i)$.

According to extensive experiment results, the precorrection algorithm can effectively reduce ranging errors 
caused by environmental factors. At the initial stage of localization, there may be only few anchor nodes available in the networks, pre-correction algorithm will not work well. However, as more nodes being localized, more anchor nodes can be identified and the precorrection algorithm will become more beneficial.

Overall, the pre-correction process first calculates the LQI difference $\left\{\delta_{i}\right\}$ between measured LQIs and those from the empirical regression function. Then, either unified or grouped correction is used to correct the measured $L Q I_{i}$ so that the errors between measured and historical LQI are minimized.

\subsection{Error Compensation}

Although the pre-correction algorithm eliminates environmental impact on LQI data, intrinsic errors may exist in LQI data due to several other factors including unknown software defects, hardware noise, issues in communication protocols, etc. In this section, we first analyze the intrinsic errors existing in LQI data and then discuss an approach to deduct these errors.

Figure 8 shows the intrinsic error curve of the regression function we used. Similar curve exist in LQI data from all experiments, so we believe the errors are caused by hardware and/or software, and always appear in LQI data. Coupling with regression function shown in Figure 4, the intrinsic errors increase when the LQI data decline quickly, e.g. around $4 \mathrm{~m}$ and $8 \mathrm{~m}$. Therefore, the first step is to deduct intrinsic errors from measured LQI data. The idea of compensating intrinsic errors is similar to the grouped correction strategy.

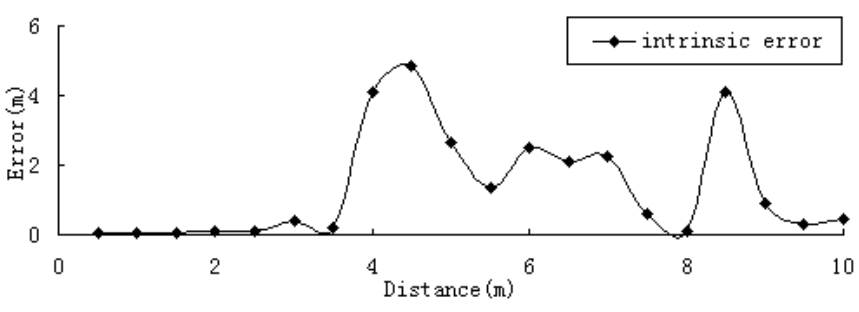

Figure 8 Illustration of Intrinsic Errors in the Empirical Regression Function

Since the empirical regression function is obtained from huge amount of historical LQI data, it is a known curve before every ranging test. However, due to the randomness in LQI data, there may be some unexpected errors in LQI which can not be handled by either the pro-correction or the error compensation processes. Therefore, the error compensation scheme can only be applied in certain conditions.

First, if compensated LQI data is not reasonable, then the LQI data before compensation is used. For instance, if the distance computed from a smaller LQI (after error compensation) is shorter than that calculated from a larger LQI, then the ranging result is questionable and should be ignored.
Second, from Figure 4 and other results, we note that LQI data decrease monotonically within $3.5 \mathrm{~m}$, i.e. the LQI data uniquely corresponds to a distance (within $3.5 \mathrm{~m}$ ). Besides, the LQI data beyond $3.5 \mathrm{~m}$ are all less than those within $3.5 \mathrm{~m}$. Therefore, if $L Q I(i)>$ $L Q I[3.5 \mathrm{~m}]$ where $L Q I[3.5 \mathrm{~m}]$ is the average LQI at $3.5 \mathrm{~m}$, then the LQI data can be use to directly estimate a distance; otherwise, it should correspond to a distance greater than $3.5 \mathrm{~m}$.

Third, if compensated LQI data corresponds to a distance which is out of the communication range, then the data before compensation will be used.

\subsection{Mixed Regression Analysis}

The pre-correction and error compensation approaches try to eliminate errors between measurement LQI curve and the empirical regression function. However, the empirical regression function may contains some errors which is modeled as $R^{2}$. to address this issue, a mixed regression analysis strategy is used to further improve the ranging accuracy. We consider four classical fitting curves: polynomial, linear, exponential and logarithmic curves. Figure 9 shows the ranging errors in different fitting curves. From 9 and several other experiments, we draw the following three conclusions.

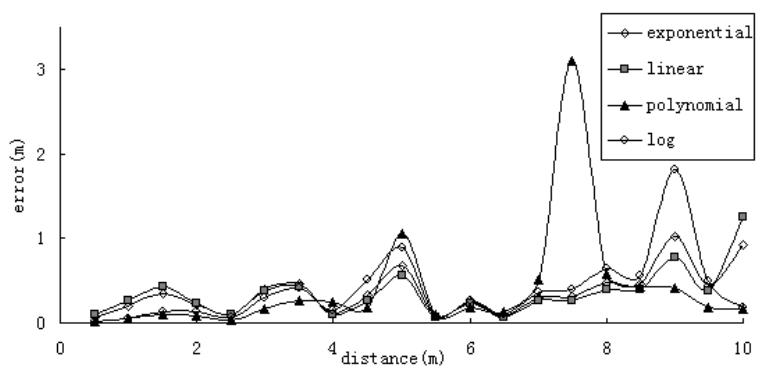

Figure 9 Average Ranging Errors of Different Fitting Curves

First, all fitting curves (polynomial, linear, exponential and logarithmic) give increasing ranging errors when distances becomes larger. All curves show similar errors at the same distance except for those at $7.5 \mathrm{~m}, 9 \mathrm{~m}$ and $10 \mathrm{~m}$.

Second, within 5m, polynomial curve give the smallest ranging error.

Third, beyond $5 \mathrm{~m}$, polynomial curve give the best ranging results except for the distance at $7.5 \mathrm{~m}$.

After analyzing the errors in four different fitting curves, we perform a mixed regression analysis in our ranging algorithm. When $L Q I(i)>L Q I[3.5 m]$, the transmitter and receiver are close to each other, and the distance between them can be calculated from polynomial regression function. When $L Q I(i)<$ $L Q I[3.5 \mathrm{~m}]$, we first compute the LQI differences $\delta$ of the four different fitting curves. Then, applying the majority rule to eliminate the fitting curve(s) that has large errors. For example, at distance $7.5 \mathrm{~m}$, polynomial regression function will be eliminated. If the majority rule does not 
applied, e.g. at distance $10 \mathrm{~m}$, then we use polynomial function as a default function.

\section{Experiments and Data Analyses}

To evaluate the accuracy of the proposed ranging techniques including pre-correction, error compensation and mixed regression analysis, we conduct another set of experiments. Experiments are conducted at both the public square of the University of Electronic Science and Technology of China (UESTC) and in the UESTC's main building.

Before the ranging tests, we first collect LQI data from 10 randomly-chosen distances. Since these LQI data are obtained from nodes with know locations, they are used in the pre-correction process. Then, we place the transmitter and receiver close to each other (distance is $0 \mathrm{~m}$ ) and move the transmitter away from the receiver with the step size of $0.5 \mathrm{~m}$. For every distance, we collect 20 LQI data and use the average LQI as the measured LQI at this distance. Overall, we have the first set of data for pre-correction and the second for ranging tests. With the help of the first set of data, group correction is applied to pre-correct the second set of data. As shown in Figure 10, the pre-corrected LQI curve matches the the regression function curve perfectly.

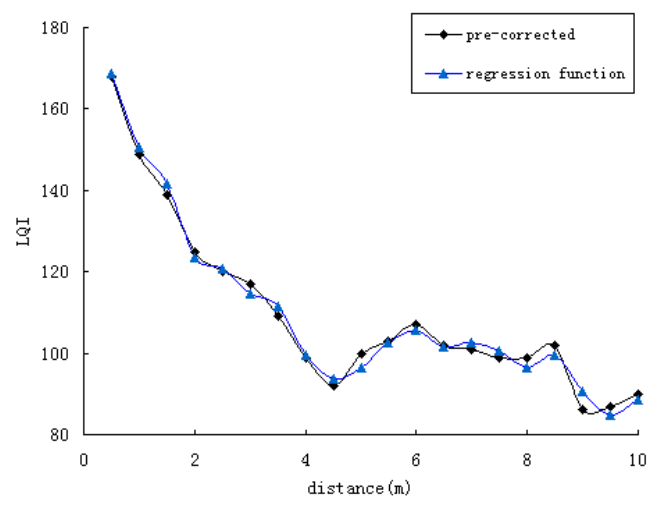

Figure 10 Comparison of Pre-Corrected and Regression Function Curves

To illustrate the necessities of all proposed techniques, we process the second set of data in four different ways. The first one uses raw LQI data and the empirical regression function to compute distances. The second one utilizes pre-correction algorithm to correct measured LQI and then substitute into the regression function to compute distance. The third one additionally apply the error compensation strategy. The last one process the data with all proposed techniques. As shown in Figure 11, the maximum ranging error of the first method is around $6.5 \mathrm{~m}$, while the errors of the last one is less than $0.9 \mathrm{~m}$. Statics summary of results from the last one is shown in Table 1.

To compare with other LQI-based ranging techniques in literature, we replicated the algorithms proposed

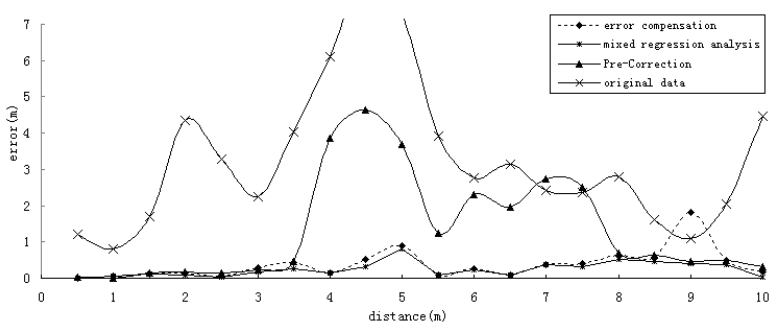

Figure 11 Comparison of Multiple Strategies

Table 1 Statistic Summary of Ranging Errors of Proposed Technique

\begin{tabular}{c|c|c|c|c}
\hline Err. $<1 m$ & Err. $<0.5 m$ & Avg. & Max. & Min. \\
\hline \hline $100 \%$ & $90 \%$ & $0.25 m$ & $0.80 m$ & $0.01 m$ \\
\hline
\end{tabular}

in (22) and (23). Table 2 summarizes the the average ranging errors for different methods. Although (23) provides a similar average ranging error as the proposed technique, it works badly when the distance is larger than 5 meters. From this table, we conclude the proposed ranging technique has better ranging accuracy.

\section{Conclusion}

From extensive experimental studies, we are positive that LQI data could be used to estimate the distances between sensors. The calculated distances from LQI data are close to the true values, except for some rare cases where LQI fluctuate severely. The overall performance of the proposed ranging technique is acceptable, i.e. with an average ranging error less than $1 \mathrm{~m}$.

After analyzing LQI data from many experiments, we discover there are huge errors in raw LQI data i.e. randomness in LQI necessitate the proposed processing techniques: pre-correction, error compensation and mixed regression analysis. Verified by experiment data, the above-mentioned techniques can effectively eliminate the errors in LQI data and significantly improve the ranging accuracy.

Although data fluctuations exist, LQI is an important metric in estimating distances because of the lost cost, low energy consumption and low requirements of hardware. Although the accuracy of the proposed LQI-based ranging techniques can be affected by physical environments, hardware and software, its performance meets the requirements of most WSN ranging/localization applications.

\section{References}

[1] Hui Liu, H. Darabi, P. Banerjee, and Jing Liu. Survey of wireless indoor positioning techniques and systems. Systems, Man, and Cybernetics, Part C: Applications and Reviews, IEEE Transactions on, 37(6):1067-1080, 2007. 
Table 2 Average Errors of Different LQI-Based Ranging Techniques

\begin{tabular}{l|l|l|l}
\hline Ranging Techniques & Average Error & Metric(s) & Environment/Range \\
\hline \hline Proposed algorithm & $0.25 \mathrm{~m}$ & LQI only & Outdoor $/ 10 \mathrm{~m}$ \\
\hline Ref $(22)$ & $1.23 \mathrm{~m}$ & RSSI only & Outdoor $/ 10 \mathrm{~m}$ \\
Dynamic Ranging & $0.86 \mathrm{~m}$ & LQI only & \\
& $0.71 \mathrm{~m}$ & LQI and RSSI & \\
\hline Ref $(23)$ & $0.24 \mathrm{~m}$ & LQI only & Indoor $/ 8 \mathrm{~m}$ \\
\hline
\end{tabular}

[2] Bram Dil, Stefan Dulman, and Paul Havinga. Range-based localization in mobile sensor networks. Wireless Sensor Networks, 3868:164-179, 2006.

[3] Nissanka B. Priyantha, Anit Chakraborty, and Hari Balakrishnan. The cricket location-support system. In Proceedings of the 6th annual international conference on Mobile computing and networking, MobiCom '00, pages 32-43, 2000.

[4] D. Niculescu and B. Nath. Ad hoc positioning system (APS) using AOA. In INFOCOM 2003. Twenty-Second Annual Joint Conference of the IEEE Computer and Communications, volume 3, pages 1734-1743, 2003.

[5] Yubin Zhao, Yuan Yang, and M. Kyas. Comparing centralized kalman filter schemes for indoor positioning in wireless sensor network. In Indoor Positioning and Indoor Navigation (IPIN), 2011 International Conference on, pages 1-10, Sept.

[6] Qing Yang Ting Yang and A. Lim. Driver layer approach to time-of-arrival ranging in IEEE 802.11g networks. In Consumer Communications and Networking Conference (CCNC), 2012 IEEE, pages 240-244, Jan.2012.

[7] Hui Liu, H. Darabi, P. Banerjee, and Jing Liu. Survey of wireless indoor positioning techniques and systems. Systems, Man, and Cybernetics, Part C: Applications and Reviews, IEEE Transactions on, 37(6):1067-1080, Nov.

[8] P. Bahl and V.N. Padmanabhan. RADAR: an in-building RF-based user location and tracking system. In INFOCOM 2000. Nineteenth Annual Joint Conference of the IEEE Computer and Communications Societies. Proceedings. IEEE, volume 2, pages 775-784 vol.2, 2000.

[9] P. Kumar, L. Reddy, and S. Varma. Distance measurement and error estimation scheme for RSSI based localization in wireless sensor networks. In Network Protocols and algorithms, 2009 Fifth IEEE Conference on, pages 1-4, 2009.

[10] Zhang Jianwu and Zhang Lu. Research on distance measurement based on rssi of zigbee. In Computing, Communication, Control, and Management, 2009. CCCM 2009. ISECS International Colloquium on, volume 3, pages 210-212, 2009.
[11] Zheng Zhang, Guocheng Wan, Min Jiang, and Guangyou Yang. Research of an adjacent correction positioning algorithm based on rssi-distance measurement. In Fuzzy Systems and Knowledge Discovery (FSKD), 2011 Eighth International Conference on, volume 4, pages 2319-2323, 2011.

[12] V. Daiya, J. Ebenezer, S.A.V.S. Murty, and Baldev Raj. Experimental analysis of rssi for distance and position estimation. In Recent Trends in Information Technology (ICRTIT), 2011 International Conference on, pages 1093-1098, 2011.

[13] A. Faheem, R. Virrankoski, and M. Elmusrati. Improving rssi based distance estimation for 802.15.4 wireless sensor networks. In Wireless Information Technology and Systems (ICWITS), 2010 IEEE International Conference on, pages 1-4, 2010.

[14] K. Benkic, M. Malajner, P. Planinsic, and Z. Cucej. Using rssi value for distance estimation in wireless sensor networks based on zigbee. In Systems, Signals and Image Processing, 2008. IWSSIP 2008. 15th International Conference on, pages 303-306, 2008.

[15] G. Gracioli, A.A. Frohlich, R. P. Pires, and L. Wanner. Evaluation of an rssi-based location algorithm for wireless sensor networks. Latin America Transactions, IEEE (Revista IEEE America Latina), 9(1):830-835, 2011.

[16] A. Awad, T. Frunzke, and F. Dressler. Adaptive distance estimation and localization in wsn using rssi measures. In Digital System Design Architectures, Methods and Tools, 200\%. DSD $200 \%$. 10th Euromicro Conference on, pages 471-478, 2007.

[17] S. Gansemer, U. Grossmann, and S. Hakobyan. Rssi-based euclidean distance algorithm for indoor positioning adapted for the use in dynamically changing wlan environments and multi-level buildings. In Indoor Positioning and Indoor Navigation (IPIN), 2010 International Conference on, pages 1-6, 2010.

[18] Monique Becker, Andre-Luc Beylot, Riadh Dhaou, Ashish Gupta, Rahim Kacimi, and Michel Marot. Experimental study: Link quality and deployment 
issues in wireless sensor networks. In Proceedings of the 8th International IFIP-TC 6 Networking Conference, NETWORKING '09, pages 14-25. Springer-Verlag, 2009.

[19] Q. Hu, Q. Zeng, F. Zhao, and Y. Liang. Research on LQI location algorithm and errors for wireless sensor networks. Journal of Chinese Computer Systems, 32(1):95-98, 2011.

[20] M. Raju, T. Oliveira, and D.P. Agrawal. A practical distance estimator through distributed RSSI/LQI processing - an experimental study. In Communications (ICC), 2012 IEEE International Conference on, pages 6575-6579, 2012.

[21] T. Oliveira and D.P. Raju, M.and Agrawal. Accurate distance estimation using fuzzy based combined RSSI/LQI values in an indoor scenario: Experimental verification. Special Issue on and smart Communication Protocols and Algorithms on Network Protocols and algorithms, 4(4):174-199, 2012.

[22] Wang Xia Zhang Jieying, Sun Maohang. Dynamic distance estimation method based on rssi and lqi. Electronic Measurement Technology, 30(2):142-145, 2007.

[23] Chu Hongyan Zhu Qianjie. Experimental analysis of nodes distance measurement based on lqi. Journal of Nanjing Normal University(Engineering and Technology Edition, 10(3):40-43, 2010. 